\title{
Impact of fermionic operators on the Higgs boson width measurement
}

\author{
Eduardo da Silva Almeida* and O. J. P. Éboli $\odot^{\dagger}$ \\ Instituto de Física, Universidade de São Paulo, São Paulo-SP 05508-090, Brazil \\ M. C. Gonzalez-Garcia \\ Institució Catalana de Recerca i Estudis Avançats (ICREA), Departament d'Estructura i Constituents de la \\ Matèria, Universitat de Barcelona, 647 Diagonal, E-08028 Barcelona, Spain \\ and C.N. Yang Institute for Theoretical Physics, SUNY at Stony Brook, Stony Brook, \\ New York 11794-3840, USA
}

(Received 6 August 2020; accepted 5 November 2020; published 8 December 2020)

\begin{abstract}
The off-shell Higgs production in $p p \rightarrow Z Z$ at LHC provides at present the most direct measurement of the Higgs width in the absence of beyond the standard model contributions. Here, we analyze the impact of anomalous $Z$ couplings to fermions on the Higgs width determination. We show that, despite these couplings being strongly constrained by the available electroweak precision data, they can substantially affect the Higgs width determination at the LHC Runs 2 and 3. Conversely, in larger integrated luminosities runs, such as those foreseen at the high luminosity LHC and high energy LHC setups, the effect of such anomalous interactions in the Higgs width measurement is minimal.
\end{abstract}

DOI: 10.1103/PhysRevD.102.113002

\section{INTRODUCTION}

In the Standard Model (SM), the Higgs boson is a relatively narrow resonance with a width $\Gamma_{H}^{\mathrm{SM}} \simeq 4.1 \mathrm{MeV}$ which is difficult to measure directly at the Large Hadron Collider (LHC). Notwithstanding, the off-shell Higgs production is sizable [1] with its tail being independent of the Higgs width. Therefore, the comparison of the onshell Higgs production, which is inversely proportional to the Higgs width, with the off-shell one allows the extraction of the Higgs width [2-4]. This procedure assumes that the off-shell Higgs production in $p p \rightarrow Z Z$ receives no contribution from new physics. In fact, the LHC experimental collaborations use this method to perform such measurement [5-8].

One characteristic feature of the off-shell Higgs production $p p \rightarrow Z Z$, is that the Higgs contribution $(g g \rightarrow H \rightarrow$ $Z Z$ ) interferes destructively with the continuum $g g \rightarrow Z Z$ generated via box diagrams [1,3,9-11]. This cancellation is affected if new physics contributes to the off-shell Higgs production, either by altering the Higgs couplings or by introducing new particles running in the loops or being

\footnotetext{
*eduardo.silva.almeida@usp.br †eboli@if.usp.br

*concha@insti.physics.sunysb.edu
}

Published by the American Physical Society under the terms of the Creative Commons Attribution 4.0 International license. Further distribution of this work must maintain attribution to the author(s) and the published article's title, journal citation, and DOI. Funded by SCOAP ${ }^{3}$. exchanged. This fact has been exploited in the literature to study departures of the Higgs couplings from their SM value, as well for signals of new physics [12-22]. In fact, the offshell Higgs production data is already being used by the experimental collaborations to probe the $H V V$ coupling and to access the impact of such anomalous Higgs interactions in the determination of its width [8].

It is also a fact that the off-shell Higgs production possesses a large background stemming from $q \bar{q} \rightarrow Z Z$ that is much larger than the off-shell Higgs signal. Consequently, changes in this background process induced, for example, by anomalous $Z \bar{q} q$ interactions have the potential to affect the determination of the Higgs width and of possible anomalous Higgs interactions. This reaction is similar to $q \bar{q} \rightarrow W^{+} W^{-} / W^{ \pm} Z$ used to probe the triple electroweak gauge couplings (TGC). And as it has been shown [23-26] that, despite the precise determination of the electroweak vector boson couplings to fermions from electroweak precision data (EWPD), anomalous fermionic couplings can impact the determination of TGC due to the present and future precisions of the LHC. It is then a matter of concern, the possible impact of $Z \bar{q} q$ anomalous interactions in the present and new future determinations of the Higgs width through the study of the off-shell Higgs production. It is the goal of this work to quantify such impact.

To this end, in this work we scrutinize the determination of the Higgs width through off-shell Higgs production taking into account anomalous $Z \bar{q} q$ couplings by analyzing the process 


$$
p p \rightarrow \ell^{+} \ell^{-} \ell^{+} \ell^{-} q
$$

where $\ell$ stands for $e$ and $\mu$. We perform our analyses for the LHC Runs 2 and 3, as well as, for the high-luminosity LHC (HL-LHC) and high-energy LHC (HE-LHC). Our results show that the presence of anomalous $Z \bar{q} q$ contributions to Eq. (1) which are not accounted for in the model to be fitted, translates both in changes in the apparent precision in the Higgs width determination in the LHC Runs 2 and 3, as well as into a shift in its derived central value. Conversely, the expected larger integrated luminosities at the HL-LHC and HE-LHC runs should be enough to mitigate these effects, making the determination of the Higgs width in those setups robust under the presence of anomalous $Z$ couplings within its present bounds.

\section{ANALYSES FRAMEWORK}

We parametrize the effects of new physics on the $Z$ couplings to quarks at low energies by dimension-six effective operators where the $S U(2)_{L} \otimes U(1)_{Y}$ gauge symmetry is linearly realized [27-33], that is,

$$
\mathcal{L}_{\text {eff }}=\mathcal{L}_{\mathrm{SM}}+\sum_{i} \frac{f_{i}^{(i)}}{\Lambda^{2}} \mathcal{O}_{i}^{(6)},
$$

where the dimension-six operators $\mathcal{O}_{i}^{(6)}$ involve gauge bosons, Higgs doublets, fermionic fields, and covariant derivatives of these fields. Here, we considered the following operators that modify the left- and right-handed couplings of the $Z$ to quarks

$$
\begin{aligned}
& \mathcal{O}_{\phi Q, i j}^{(1)}=\phi^{\dagger}\left(i \stackrel{\leftrightarrow}{D_{\mu}} \phi\right)\left(\bar{Q}_{i} \gamma^{\mu} Q_{j}\right), \\
& \mathcal{O}_{\phi Q, i j}^{(3)}=\phi^{\dagger}\left(i \stackrel{\leftrightarrow}{D}{ }_{\mu} \phi\right)\left(\bar{Q}_{i} \gamma^{\mu} T_{a} Q_{j}\right), \\
& \mathcal{O}_{\phi u, i j}^{(1)}=\phi^{\dagger}\left(i \stackrel{\leftrightarrow}{D}_{\mu} \phi\right)\left(\bar{u}_{R_{i}} \gamma^{\mu} u_{R_{j}}\right), \\
& \mathcal{O}_{\phi d, i j}^{(1)}=\phi^{\dagger}\left(i \stackrel{\leftrightarrow}{D}_{\mu} \phi\right)\left(\bar{d}_{R_{i}} \gamma^{\mu} d_{R_{j}}\right),
\end{aligned}
$$

where we defined $\tilde{\phi}=i \sigma_{2} \phi^{*}, \phi^{\dagger} \stackrel{\leftrightarrow}{D}_{\mu} \phi=\phi^{\dagger} D_{\mu} \phi-\left(D_{\mu} \phi\right)^{\dagger} \phi$ and $\phi^{\dagger} \stackrel{\leftrightarrow}{D}_{\mu}^{a} \phi=\phi^{\dagger} T^{a} D_{\mu} \phi-\left(D_{\mu} \phi\right)^{\dagger} T^{a} \phi$ with $T^{a}=\sigma^{a} / 2$ and $\sigma^{a}$ standing for the Pauli matrices. We have also used the notation of $Q$ for the quark doublet and $f_{R}$ for the $S U(2)_{L}$ singlet quarks. Here, $i, j$ are family indices and, for the sake of simplicity, we consider only diagonal family couplings that are generation independent.

In addition to the above anomalous couplings, we also considered the electroweak dipole operators

$$
\begin{array}{ll}
\mathcal{O}_{u W, i j}=i \bar{Q}_{i} \sigma^{\mu \nu} u_{R, j} \hat{W}_{\mu \nu} \tilde{\phi}, & \mathcal{O}_{u B, i j}=i \bar{Q}_{i} \sigma^{\mu \nu} u_{R, j} \hat{B}_{\mu \nu} \tilde{\phi}, \\
\mathcal{O}_{d W, i j}=i \bar{Q}_{i} \sigma^{\mu \nu} d_{R, j} \hat{W}_{\mu \nu} \phi, & \mathcal{O}_{d B, i j}=i \bar{Q}_{i} \sigma^{\mu \nu} u_{R, j} \hat{B}_{\mu \nu} \phi,
\end{array}
$$

TABLE I. 95\% C.L. allowed regions for the Wilson coefficients $f / \Lambda^{2}$ in $\mathrm{TeV}^{-2}$ taking into account the electroweak precision data and diboson production $[25,34]$.

\begin{tabular}{lcc}
\hline \hline Operator & EWPD & EWPD + EWDBD \\
\hline $\mathcal{O}_{\phi Q}^{(1)}$ & {$[-0.083,0.10]$} & {$[-0.034,0.11]$} \\
$\mathcal{O}_{\phi Q}^{(3)}$ & {$[-0.60,0.12]$} & {$[-0.45,0.13]$} \\
$\mathcal{O}_{\phi d}^{(1)}$ & {$[-1.2,-0.13]$} & {$[-0.64,-0.007]$} \\
$\mathcal{O}_{\phi u}^{(1)}$ & {$[-0.25,0.37]$} & {$[-0.17,0.37]$} \\
$\mathcal{O}_{u W}$ & {$[-10,10]$} & {$[-0.29,0.29]$} \\
$\mathcal{O}_{u B}$ & {$[-41,41]$} & {$[-1.9,1.9]$} \\
$\mathcal{O}_{d W}$ & {$[-10,10]$} & {$[-0.36,0.36]$} \\
$\mathcal{O}_{d B}$ & {$[-38,38]$} & {$[-1.9,1.9]$} \\
\hline \hline
\end{tabular}

where we defined $\hat{B}_{\mu \nu} \equiv i\left(g^{\prime} / 2\right) B_{\mu \nu}$ and $\hat{W}_{\mu \nu} \equiv i(g / 2) \sigma^{a} W_{\mu \nu}^{a}$, with $g$ and $g^{\prime}$ being the $S U(2)_{L}$ and $U(1)_{Y}$ gauge couplings respectively.

Presently, the Wilson coefficients of the dimension-six operators in Eqs. (3) and (4) are bound by EWPD and electroweak diboson data (EWDBD) [25,34-37]. For convenience, in Table I, we summarize these limits as derived in Refs. [25,34]. As seen in this table they are roughly of the order of $1 \mathrm{TeV}^{-2}$. Future $\mathrm{LHC}$ runs have the potential to improve these constraints. Preliminary results of global fits indicate that the HL- and HE-LHC datasets will have little impact on the precision of the Wilson coefficients of $\mathcal{O}_{\phi Q}^{(1)}, \mathcal{O}_{\phi d}^{(1)}$ and $\mathcal{O}_{\phi u}^{(1)}$, however, the precision on the Wilson coefficient of $\mathcal{O}_{\phi Q}^{(3)}$ will improve by a factor of 3; see Fig. 136 of Ref. [38].

As mentioned above, we are assuming the coefficients to be generation independent. For the effects discussed in this paper which involve couplings to light quarks, the constraints on the Wilson coefficients for these operators will be relaxed at most by factor $\mathcal{O}(3)$ if one allows for the couplings to be different for the third generation quarks [39]. Of course in this case, the couplings to the top quark are allowed to be larger and can lead to larger effects in the gluon-fusion processes to the off-shell Higgs signal; we return to this point at the end of this section.

Generically, in order to extract the Higgs width from offshell Higgs data, the gluon-gluon cross section is parametrized as

$$
\sigma\left(g g \rightarrow \ell^{+} \ell^{-} \ell^{+} \ell^{-}\right)=\sigma_{\text {cont }}+\sqrt{X} \sigma_{\text {inter }}+X \sigma_{H},
$$

where the coefficient

$$
X=\mu_{4 \ell} \times \frac{\Gamma_{H}}{\Gamma_{H}^{\mathrm{SM}}}
$$

is the variable to be determined from the analysis. $\Gamma_{H}$ and $\Gamma_{H}^{\mathrm{SM}}$ stand for the Higgs total width to be determined and its SM predicted value respectively. $\mu_{4 \ell}$ is the signal strength 
for the Higgs production in the four-lepton channel which can be independently constrained by on-shell Higgs data.

We denoted by $\sigma_{\text {cont }}$ the continuum cross section, by $\sigma_{H}$ the Higgs contribution and by $\sigma_{\text {inter }}$ the interference between continuum and Higgs contributions. In our simulations these gluon-gluon initiated contributions were evaluated using MCFM-9.1 [3]. In our calculations, we used the NNPDF 3.0 parton distribution functions [40] in LHAPDF 6.2.3 [41].

An irreducible background to the above process is induced at tree level by

$$
q \bar{q} \rightarrow \ell^{+} \ell^{-} \ell^{+} \ell^{-} .
$$

In order to evaluate the contributions of the above anomalous $Z \bar{q} q$ couplings to the process in Eq. (7), we used the package FeynRules [42] to generate the UFO files needed as input into MadGraph 5 [43], that we employed to compute the tree level cross sections.

Finally, let us notice that the anomalous $Z \bar{q} q$ couplings also contribute to the box diagram $g g \rightarrow Z Z$, therefore, it modifies the continuum and interference cross sections. Indeed the anomalous $Z \bar{t} t$ anomalous coupling contributions have already been considered when studying new-physics effects in off-shell Higgs production; see for instance Ref. [20]. However, the effect os anomalous $Z$ couplings to the light quarks has not been analyzed in the $q \bar{q}$ process, neither in the gluon-gluon loops. We took into account this effect for the couplings in Eq. (3) to the lowest order in $1 / \Lambda^{2}$ and it was evaluated using a modified version of MCFM-9.1.

\section{RESULTS}

In what follows we are going to use as main observable the four-lepton invariant mass distribution of events. To do so, we performed a parton level simulation and we imposed cuts similar to the ones in Ref. [8], that is, we required the final state leptons to satisfy

$p_{T}^{\ell}>10 \mathrm{GeV}, \quad\left|\eta_{\ell}\right|<2.4, \quad p_{T, \text { hardest }}^{\ell}>20 \mathrm{GeV}$

and that the events should exhibit two same-flavor opposite charge lepton pair close to the $Z$ mass, i.e.,

$$
40<m_{\ell \ell}<120 \mathrm{GeV} .
$$

We analyzed four setups: for the LHC Run2 (Run3/HL) we considered a center-of-mass energy of 13 (14) $\mathrm{TeV}$ and an integrated luminosity of $140(400 / 3000) \mathrm{fb}^{-1}$, while for the HE-LHC we assumed a center-of-mass energy of $27 \mathrm{TeV}$ and an integrated luminosity of $15 \mathrm{ab}^{-1}$.

In Fig. 1, we depict the resulting four-lepton invariant mass distributions for the center-of-mass energies of $13 \mathrm{TeV}$ and $27 \mathrm{TeV}$; the results for $14 \mathrm{TeV}$ are similar to the $13 \mathrm{TeV}$ ones. In the top two panels we display the SM expected distributions, as well as the quark-antiquark fusion contribution from the dipole operator $\mathcal{O}_{d W}$ (more below). In the middle and lower panels of Fig. 1, we present the $1 / \Lambda^{2}$ anomalous contributions of the operators in Eq. (3) for the quark- and gluon-fusion channels. All the anomalous cross sections shown have been evaluated for a Wilson coefficient $f / \Lambda^{2}=1 \mathrm{TeV}^{-2}$. Amplitudes induced by the operators in Eq. (3) interfere with the SM ones and the cross sections have been evaluated to linear order in the corresponding Wilson coefficient. Indeed, within the present bounds in Table I this is the dominant contribution. On the contrary, amplitudes induced by dipole operators do not interfere with the SM one and therefore the cross sections shown are quadratic in the corresponding Wilson coefficient. $^{1}$

From this figure we see that quark-antiquark fusion cross sections induced by some of the operators in Eq. (3) are quantitatively comparable in size and shape to those of the SM Higgs induced subprocesses. Consequently, if these contributions are present in the data but not included in the analysis they can alter the extracted value of the $X$ coefficient in Eq. (6). Quantitatively, within the present bounds on the Wilson coefficients, we find that the largest potential effect would correspond to $\mathcal{O}_{\phi Q}^{(3)}$ in which we focus in the following.

Conversely, from the upper panels of Fig. 1 we learn that anomalous dipole contributions exhibit a spectrum different than the SM one with the dipole contribution becoming more important at high four-lepton invariant masses. This is expected since dipole scattering amplitude grows as $m_{4 \ell}^{2}$ at high energies [39]. This, in principle, makes the effect of these operators easier to disentangle from a shift in the Higgs width. However, as we will show below, this relies on having enough statistics in the larger invariant mass bins. Let us point out that the results shown are for $\mathcal{O}_{d W}$ but similar results are obtained for $\mathcal{O}_{d B}, \mathcal{O}_{u W}$, and $\mathcal{O}_{u B}$. In particular, as the amplitudes induced by these operators do not interfere with the SM amplitudes, the contributions from each of these operators are independent from each other at quadratic order in their coefficients. In particular results for $\mathcal{O}_{d B}$ are very close from those shown rescaled down by the corresponding ratio of coefficients of the induced dipole $Z q q$ coupling $\left(f_{d W} \rightarrow s_{W}^{2} / c_{W}^{2} f_{u W}\right)$. Conversely for couplings to the $u$ quark the differences in the quark distribution functions make the corresponding corrections slightly larger.

To further illustrate and quantify the impact of the anomalous $Z \bar{q} q$ couplings in the Higgs width determination, we show in Fig. 2 the relative change in the number of events induced by the anomalous coupling compared to that induced by a shift of the $X$ coefficient

$$
\frac{N_{D}-N_{X}}{N_{D}}
$$

\footnotetext{
${ }^{1}$ Notice that we have only considered the effects of the dipole operators to quark-fusion processes since their effects in gluon initiated processes are not available yet.
} 

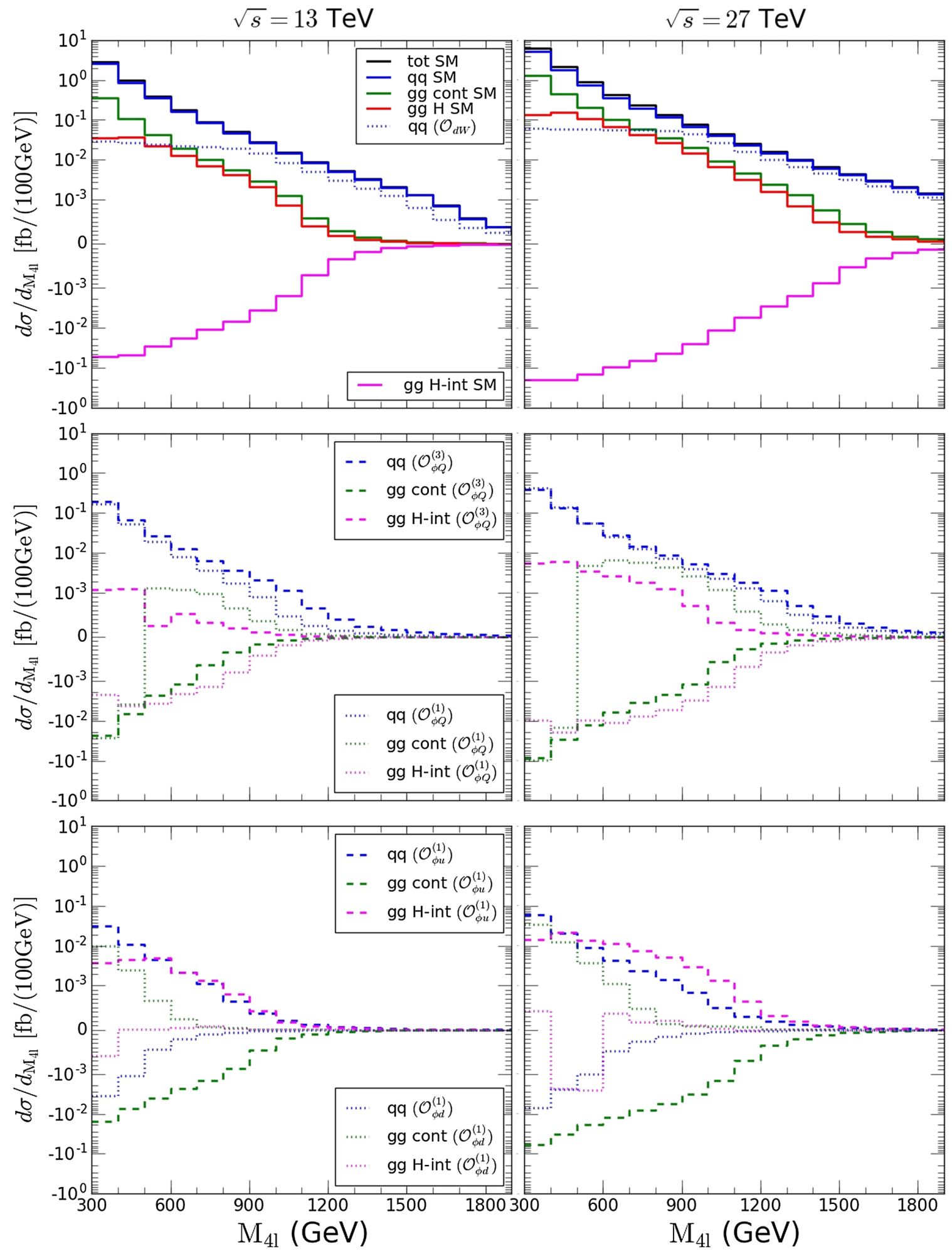

FIG. 1. Four-lepton invariant mass distributions for the different processes relevant to our study as labeled in the figure. All anomalous contributions have been computed for a Wilson coefficient $f / \Lambda^{2}=1 \mathrm{TeV}^{-2}$.

where by $N_{D}$ we denote the expected number of events considering the SM $q \bar{q}$ and $g g$ continuum contributions, as well as the one due to the $Z$ anomalous coupling of coefficient $f$, this is

$$
N_{D}=\mathcal{L} \times\left[\sigma_{q \bar{q}}^{\mathrm{SM}}+\sigma_{q \bar{q}}^{a n o}(f)+\sigma_{g g}(X=1, f)\right],
$$

where $\mathcal{L}$ is the integrated luminosity. $N_{X}$ stands for the number of events expected from SM $q \bar{q}$ and $g g$ continuum 

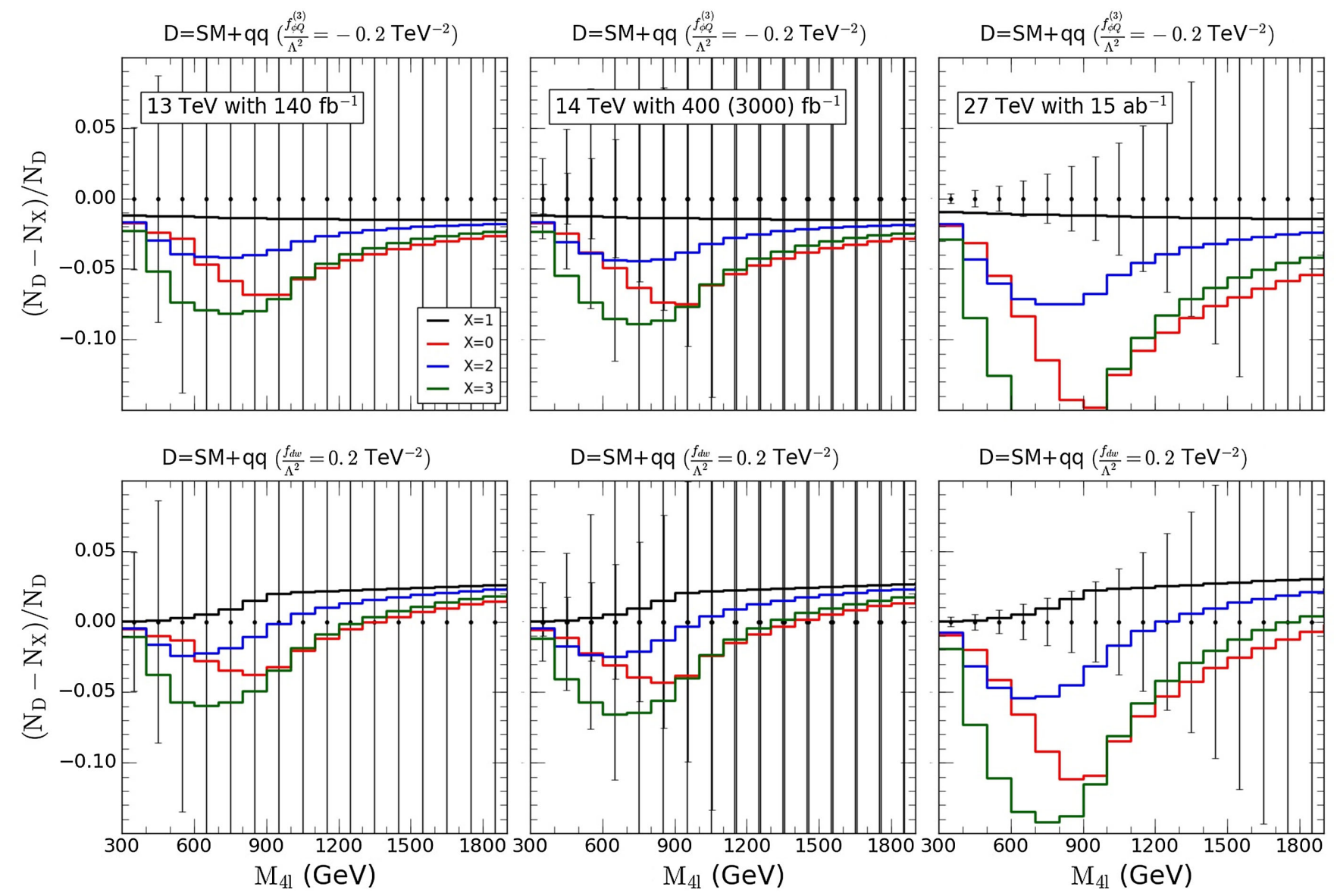

FIG. 2. Relative variation of the expected number of event distribution as induced by anomalous $Z \bar{q} q$ couplings versus a nonstandard Higgs width coefficient $X$. In the upper (lower) panels $f_{\phi Q}^{(3)} / \Lambda^{2}=-0.2 \mathrm{TeV}^{2}\left(f_{d W} / \Lambda^{2}=0.2 \mathrm{TeV}^{2}\right)$. The left, middle, and right panels corresponds to the LHC Run2, Run3/HL, and HE-LHC respectively. For comparison, we show as error bars the corresponding statistical uncertainties for an hypothetical observation according to $N_{D}$. In the central panel the larger (smaller) error bars correspond to $\mathcal{L}=400(3000) \mathrm{fb}^{-1}$.

contributions together with the Higgs one with a different value of the coefficient $X$; see Eq. (5).

$$
N_{X}=\mathcal{L} \times\left[\sigma_{q \bar{q}}^{\mathrm{SM}}+\sigma_{g g}(X, f=0)\right]
$$

The upper panels correspond to $f=f_{\phi Q}^{(3)} / \Lambda^{2}=-0.2 \mathrm{TeV}^{2}$ and the lower panels to $f_{d W} / \Lambda^{2}=0.2 \mathrm{TeV}^{2}$, values well within their presently allowed $2 \sigma$ range. The left, middle and right panels correspond to the LHC Run2, Run3/HL, and HE-LHC respectively. For comparison we show as error bars the corresponding statistical uncertainties for an hypothetical observation according to $N_{D}$.

As we can see, the largest statistical weight originates from invariant-mass bins smaller than 500 (700/900/ 1200) GeV for the LHC Run 2 (LHC Run 3/HL-LHC/ HE-LHC). It is interesting to notice that the growth of the anomalous cross sections at high four-lepton invariant masses for the dipole operator $\mathcal{O}_{d W}^{(3)}$ turns out not to be very statistically significant.

Altogether Fig. 2 clearly illustrates that with the LHC Run2 and Run3 statistics the effect of the anomalous $q \bar{q}$ background and that of an anomalous Higgs width cannot be disentangled even for the dipolelike operator.

In order to estimate the quantitative effect on the determination of the Higgs width, we perform a fit to the invariant mass event distribution to extract the coefficient $X$ under the assumption that Nature contains a SM Higgs but it also has anomalous $Z \bar{q} q$ couplings. So in each bin $j, N_{\text {data }}^{j}$ follows the predicted number of events in Eq. (10). We then fit that data to a model with SM background and a Higgs signal with a width to be determined, i.e., we use $N_{\text {model }}^{j}(X)$ as Eq. (11).

With these numbers of expected and data events we construct a binned-likelihood $\chi^{2}$ for the four-lepton invariant mass distribution using $100 \mathrm{GeV}$ bins that start at $300 \mathrm{GeV}$ : 


$$
\chi^{2}(X)=2 \min _{\xi}\left\{\sum_{j=\text { bins }}\left[(1+\xi) N_{\text {model }}^{j}(X)-N_{\text {data }}^{j}+N_{\text {data }}^{j} \ln \frac{N_{\text {data }}^{j}}{(1+\xi) N_{\text {model }}^{j}(X)}\right]+\frac{\xi^{2}}{\delta_{\xi}^{2}}\right\}
$$

In constructing Eq. (12) we have introduced the $\xi$ pull to account for the possible effect of systematic uncertainties in the overall normalization and to have a rough estimate of the impact of these uncertainties. The LHC Run 2 luminosity uncertainty is of the order of $2.5 \%$ and it is expected to be reduced to $1 \%$ for the HL-LHC [38]. In addition, the LHC collaborations estimate that the uncertainty due to the choice of the QCD scales amounts to $\simeq 3-5 \%[6,8]$.

It is important to stress that the analysis performed by the experimental collaborations to extract the Higgs width make use of much more sophisticated variables [5-8] which cannot be implemented in our simplified simulation. Still, the most sensitive physics variable to the Higgs width is the 4-lepton invariant mass dependence of the different contributions. So, even if it is clear that our study cannot reproduce the absolute precision in the width determination of the experimental analysis, it should be good enough to quantify its relative change due to the presence of the anomalous $Z \bar{q} q$ couplings.

The dependence of the quality of the fit with the value of the $Z \bar{q} q$ anomalous coupling used in the generation of the data is shown in Fig. 3. The left upper panel displays the minimum $\chi^{2}$ as a function of the $f_{\phi Q}^{(3)} / \Lambda^{2}$ for the different setups. For each setup we show the results for two analyses, one in which only statistical uncertainties are considered (dashed lines) and another including the effect of an overall normalization systematic (full lines). As we can see, in the unrealistic case of only having statistical errors, the quality of the fit is not good for the HL-LHC and HE-LHC unless the anomalous coupling is rather small. However, when the
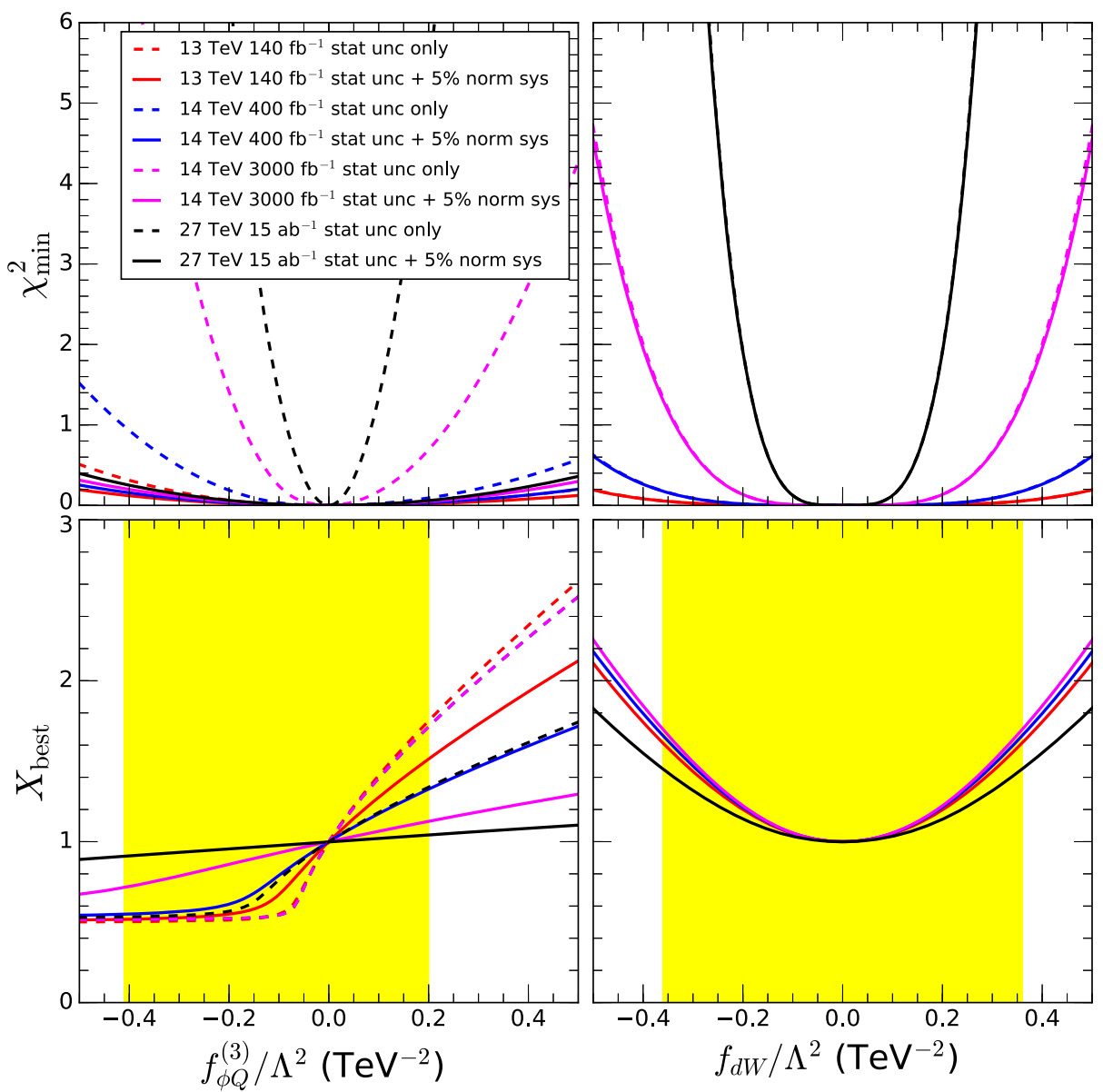

FIG. 3. The upper (lower) panels display the minimum $\chi^{2}$ (best fit $X$ ) as a function of the Wilson coefficient $f_{\phi Q}^{(3)} / \Lambda^{2}($ left) and $f_{d W}^{(3)} / \Lambda^{2}$ (right). The presently allowed regions for these couplings are indicated by the yellow bands. The different center-of-mass energies and integrated luminosities follow the color code indicated in the figure. The dashed lines correspond to fits with no normalization uncertainty while the full lines correspond to the fit including a normalization uncertainty $\delta_{\xi}=5 \%$. 
systematic normalization error is included we find a satisfactory fit for all range of anomalous couplings considered.

In the left lower panel of Fig. 3 we plot the value of the corresponding $X$ coefficient which leads to the best fit $\left(X_{\text {best }}\right)$ for the analysis including the normalization systematic uncertainty. Obviously, as the data is assumed to contain always a SM Higgs, the best fit for zero anomalous $Z \bar{q} q$ couplings is always at $X_{\text {best }}=1$. But, as seen in this figure, for the LHC Run2 (Run3) $X_{\text {best }}$ deviates considerably from the SM value $X=1$ when the anomalous $Z \bar{q} q$ contribution is included. In fact, this is the case in a sizable fraction of the allowed range of this anomalous coupling. This suggests that the limits on $\Gamma_{H}$ are affected in the presence of the anomalous interactions while the global quality of the fit is not. On the contrary, as the integrated luminosity increases for the HL-LHC and HELHC, $X_{\text {best }}$ remains around the SM value for all presently allowed values of the anomalous coupling. We notice in passing that the shift induced in $X_{\text {best }}$ from the SM value of 1 is negative (positive) for negative (positive) value of $f_{\phi Q}^{(3)} / \Lambda^{2}$. This is expected as the dominant $1 / \Lambda^{2}$ contribution is that induced on the $q \bar{q}$ background, that has the same sign as $f_{\phi Q}^{(3)} / \Lambda^{2}$. On the other hand the contribution due to the gluon-gluon processes, which has the opposite sign, is always subdominant; see dashed lines in middle panels of Fig. 1.

The corresponding results for the dipole operator coupling $f_{d W}^{(3)} / \Lambda^{2}$ are shown in the right panels of Fig. 3. In this case we find that the inclusion of the systematic normalization uncertainty has no effect in the quality of the fit. This is so because the invariant mass dependence of the anomalous $Z \bar{q} q$ events is sufficiently different from those due to the Higgs exchange processes; see upper panels in Fig. 1. Consequently, the change induced in the $m_{4 l}$ distribution of the number of events cannot be fitted with a modified $X$ coefficient even if the prediction in all bins could be globally shifted by an overall normalization factor. The dependence of $X_{\text {best }}$ upon $f_{d W}^{(3)} / \Lambda^{2}$ is shown in the right lower panel of this figure from which we see, that for couplings close to the limits of the presently allowed region, $X_{\text {best }}$ also deviates noticeably from SM value of 1 . Notwithstanding, the effect for the HL-LHC and HE-LHC is only important for values of these couplings for which the fit is never good. Finally, as expected, in this case the shift in $X_{\text {best }}$ with respect to 1 is always positive irrespective of the sign of the coupling because the anomalous contribution is positive and quadratic in $f_{d W}^{(3)} / \Lambda^{2}$.

Finally we study the impact of $Z$ anomalous coupling on the apparent precision of the measurement of the Higgs width. As mentioned above with our simplified analysis we cannot reproduce the precision attainable in dedicated experimental studies. But we can still estimate how the presence of the anomalous $Z \bar{q} q$ coupling affects that precision. In the same fashion we can estimate how the induced modification on the precision is expected to change in the different setups.

To this end we introduce normalized $1 \sigma$ upper and lower errors as

$$
\hat{\sigma}^{ \pm} \equiv \frac{\sigma^{ \pm}(f, \sqrt{s}, \mathcal{L})}{\sigma^{ \pm}\left(0,13 \mathrm{TeV}, 140 \mathrm{fb}^{-1}\right)} .
$$

Defined this way the extracted $68 \%$ C.L. allowed range of the coefficient $X$ for SM $Z \bar{q} q$ couplings in the Run 2 setup is $X=1 \pm 1$ by construction. For the Run 2 setup, the variation of this range with the anomalous coupling quantifies the relative change on the determination of the central value and precision of Higgs width due to the presence of the anomalous $Z \bar{q} q$ coupling. For other setups it also accounts for the improvement in the determination of $X$ due to the higher luminosity and center-of-mass energy.

This is what we show in Fig. 4 where we plot the $1 \hat{\sigma}$ allowed range around $X_{\text {best }}$ as a function of the Wilson coefficients of the operators $\mathcal{O}_{\phi Q}^{(3)}$ and $\mathcal{O}_{d W}$ for the several setups for which we have found a good fit in the presence of these interactions. From this figure we see that besides a shift in the best fit value, the presence of the anomalous $Z \bar{q} q$ couplings results into a variation in the attainable precision.

For $f_{\phi Q}^{(3)} / \Lambda^{2}$ the presence of the anomalous $Z \bar{q} q$ coupling leads to a noticeable increase (reduction) in the uncertainty in the $X$ determination for positive (negative) values of this Wilson coefficient. The effect is largest at the LHC Run 2 setup (upper left panel). At the LHC Run 3, see upper central panel, the precision increases due to the larger statistics but, still, both the best fit $X$ value and its error show a non-negligible dependence on $f_{\phi Q}^{(3)} / \Lambda^{2}$. However, for the HL-LHC and HE-LHC setups (lower left and central panels), $\hat{\sigma}^{ \pm}$diminishes considerably and it becomes rather independent of the $Z$ anomalous coupling. In other words, the foreseen accumulated luminosity for these setups will allow for the measurement of the Higgs width which will be robust under the possible presence of unaccounted anomalous $Z \bar{q} q$ couplings.

The right panels in Fig. 4 show the corresponding results for the dipole operator $\mathcal{O}_{d W}$ for the LHC Run 2 and Run 3 setups. As seen in the figure the presence of a nonvanishing value of this anomalous $Z \bar{q} q$ coupling always results into an apparent less precise determination of $X$.

We finish by pointing out that, for the sake of simplicity, the results in Figs. 3 and 4 have been obtained assuming only the shown coefficient (either $f_{\phi Q}^{(3)}$ or $f_{d W}$ ) to be different from zero while setting all the undisplayed ones to zero. We have verified that the results change very little if in each panel we allow all undisplayed coefficients to vary in the off-shell Higgs analisys within the priors imposed by 

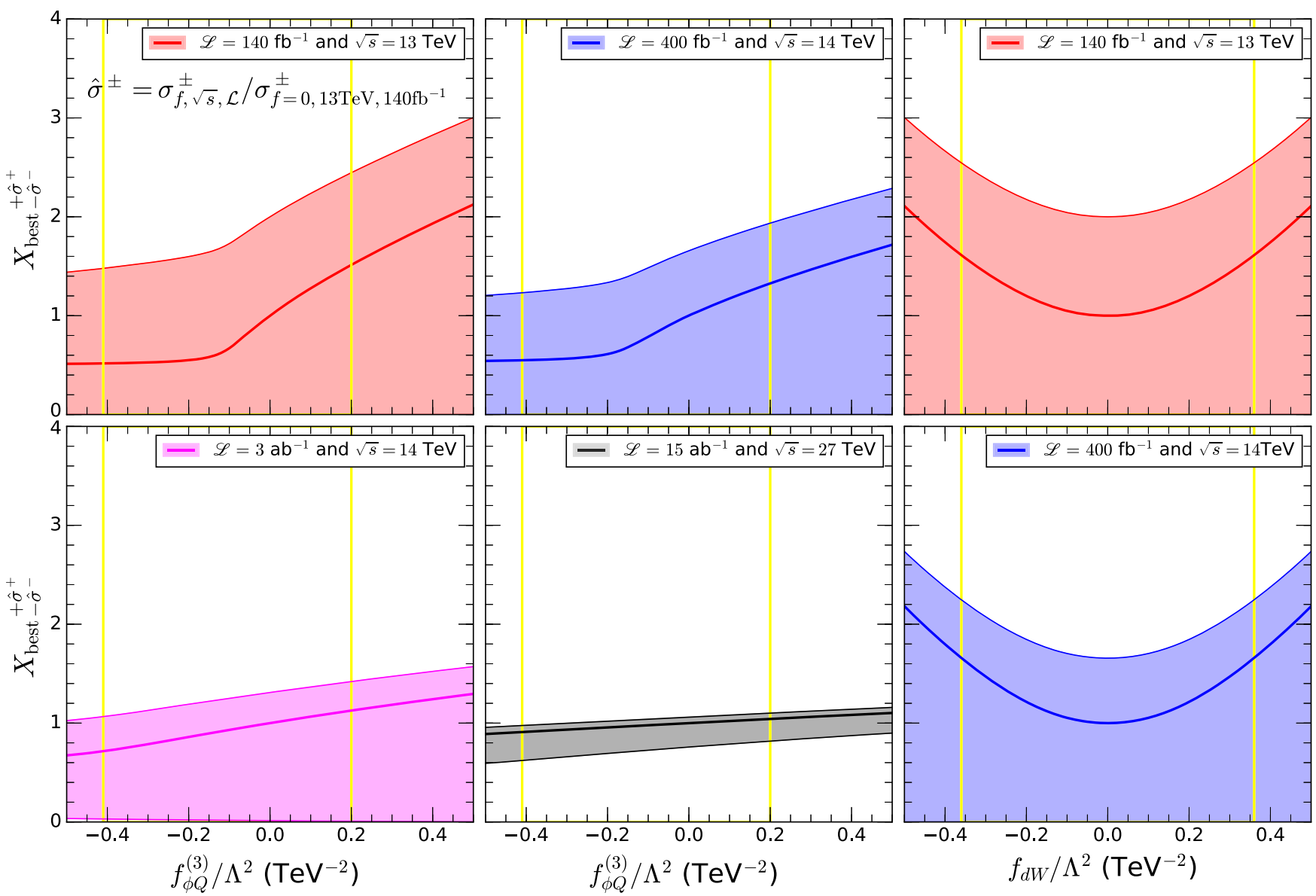

FIG. 4. Dependence of the normalized $1 \sigma$ range of the coefficient $X$ with the Wilson coefficients $f_{\phi Q}^{(3)} / \Lambda^{2}$ (left/middle panels) and $f_{d W}^{(3)} / \Lambda^{2}$ (right panels); see text for details. The presently allowed values for these couplings is indicated by the yellow vertical lines and the results are shown for different LHC setups as labeled in the figure. In all cases the fit includes a normalization uncertainty $\delta_{\xi}=5 \%$.

the constraints from electroweak precision data and diboson production data.

\section{CONCLUSIONS}

In the Standard Model, the Higgs boson is a relatively narrow resonance. At the LHC, measuring its width by profiling the direct production cross section is challenging. At present the most precise available method to directly determine the Higgs boson width at LHC is based on the study of the off-shell Higgs production in $p p \rightarrow Z Z$. This analysis makes use of the fact that the Higgs contribution, $g g \rightarrow H \rightarrow Z Z$, the continuum $g g \rightarrow Z Z$ generated via box diagrams and their interference depend on different powers of the Higgs decay width; see Eq. (5). The sensitivity of this process to $\Gamma_{H}$ is helped by the fact that, in the Standard Model, the off-shell Higgs amplitude and its interference with the continuum one give contributions of comparable size but opposite sign, so they cancel up to an amount which depends on the value of the width. For the same reason the study is sensitive to the presence of new physics which modify the relative size of these terms and as such it has been exploited in the literature.

In this work we have focused on a different aspect of this process which is associated to the presence of the irreducible background originating from $q \bar{q}$ annihilation at tree level. Being a tree level process, this background is large and, therefore, any new physics contribution to $Z \bar{q} q$ couplings, even if subdominant, can be of comparable size to that due to corrections to gluon-gluon Higgs terms from deviations of the Higgs width from the Standard Model value.

To quantify this possibility we have worked in the framework of effective Lagrangians parametrizing the effects of new physics on the $Z$ couplings to quarks at low energies by the dimension-six effective operators in Eqs. (3) and (4). In Figs. 1, and 2 we explicitly show that, despite the precise determination of the electroweak vector boson couplings to fermions from electroweak precision data, the induced anomalous fermionic couplings can give contributions to the $q \bar{q}$ background large enough to potentially affect the Higgs width extraction from fourlepton events. 
In order to estimate the possible quantitative effect of these anomalous background contributions, we have performed a statistical analysis of the four-lepton invariant mass distribution. Our assumption is that the data contain a Standard Model Higgs plus a $Z$ boson with such anomalous couplings, but that the model to be fitted has standard $Z \bar{q} q$ couplings and a Higgs with an unknown width to be determined. We have performed such analyses for the LHC Runs 2 and 3, as well as, for the highluminosity LHC and high-energy LHC. Our results are shown in Figs. 3 and 4.

We conclude that the presence of anomalous $Z \bar{q} q$ contributions to the background in the data which is not accounted for in the model to be fitted: (a) affects the apparent precision in the Higgs width determination in the LHC Runs 2 and 3, (b) induces a a shift in its derived best fit value. Our results also show that the expected larger integrated luminosities at the HL-LHC and HE-LHC runs should be enough to mitigate these effects, making the determination of the Higgs width in those setups robust under the presence of anomalous $Z$ couplings within its present bounds, even if they are ignored when performing the analysis.

\section{ACKNOWLEDGMENTS}

We thank Nuno Rosa Agostinho for his contribution in the early stages of this work. O. J.P.E. is supported in part by Conselho Nacional de Desenvolvimento Centífico e Tecnológico (CNPq) and by Fundação de Amparo à Pesquisa do Estado de São Paulo (FAPESP) Grant No. 2019/04837-9; E. S. A. thanks FAPESP for its support (Grant No. 2018/16921-1). M. C. G.-G. is supported by USA-NSF Grant No. PHY-1915093, by the spanish Grant No. FPA2016-76005-C2-1-P, and by AGAUR (Generalitat de Catalunya) Grant No. 2017-SGR-929.
[1] N. Kauer and G. Passarino, J. High Energy Phys. 08 (2012) 116.

[2] F. Caola and K. Melnikov, Phys. Rev. D 88, 054024 (2013).

[3] J. M. Campbell, R. K. Ellis, and C. Williams, J. High Energy Phys. 04 (2014) 060.

[4] J. M. Campbell, R. K. Ellis, and C. Williams, Phys. Rev. D 89, 053011 (2014).

[5] G. Aad et al. (ATLAS Collaboration), Eur. Phys. J. C 75, 335 (2015).

[6] M. Aaboud et al. (ATLAS Collaboration), Phys. Lett. B 786, 223 (2018).

[7] V. Khachatryan et al. (CMS Collaboration), J. High Energy Phys. 09 (2016) 051.

[8] A. M. Sirunyan et al. (CMS Collaboration), Phys. Rev. D 99, 112003 (2019).

[9] E. Glover and J. van der Bij, Nucl. Phys. B321, 561 (1989).

[10] T. Binoth, M. Ciccolini, N. Kauer, and M. Kramer, J. High Energy Phys. 12 (2006) 046.

[11] G. Passarino, J. High Energy Phys. 08 (2012) 146.

[12] J. S. Gainer, J. Lykken, K. T. Matchev, S. Mrenna, and M. Park, Phys. Rev. D 91, 035011 (2015).

[13] C. Englert and M. Spannowsky, Phys. Rev. D 90, 053003 (2014).

[14] G. Cacciapaglia, A. Deandrea, G. Drieu La Rochelle, and J.-B. Flament, Phys. Rev. Lett. 113, 201802 (2014).

[15] A. Azatov, C. Grojean, A. Paul, and E. Salvioni, Zh. Eksp. Teor. Fiz. 147, 410 (2015), http://www.jetp.ac.ru/cgi-bin/e/ index/r/147/3/p410?a=list.

[16] C. Englert, Y. Soreq, and M. Spannowsky, J. High Energy Phys. 05 (2015) 145.

[17] M. Buschmann, D. Goncalves, S. Kuttimalai, M. Schonherr, F. Krauss, and T. Plehn, J. High Energy Phys. 02 (2015) 038 .
[18] I. Brivio, O. Éboli, M. Gavela, M. Gonzalez-Garcia, L. Merlo, and S. Rigolin, J. High Energy Phys. 12 (2014) 004.

[19] T. Corbett, O. J. P. Éboli, D. Goncalves, J. González-Fraile, T. Plehn, and M. Rauch, J. High Energy Phys. 08 (2015) 156.

[20] A. Azatov, C. Grojean, A. Paul, and E. Salvioni, J. High Energy Phys. 09 (2016) 123.

[21] D. Gonalves, T. Han, and S. Mukhopadhyay, Phys. Rev. D 98, 015023 (2018).

[22] H.-R. He, X. Wan, and Y.-K. Wang, arXiv:1902.04756.

[23] Z. Zhang, Phys. Rev. Lett. 118, 011803 (2017).

[24] A. Alves, N. Rosa-Agostinho, O. J. Éboli, and M. GonzalezGarcia, Phys. Rev. D 98, 013006 (2018).

[25] E. da Silva Almeida, A. Alves, N. Rosa Agostinho, O. J. Éboli, and M. Gonzalez-Garcia, Phys. Rev. D 99, 033001 (2019).

[26] P. Azzi et al., Report from Working Group 1: Standard Model Physics at the HL-LHC and HE-LHC (CERN, Geneva, 2019), Vol. 7, pp. 1-220, https://doi.org/ 10.23731/CYRM-2019-007.1.

[27] W. Buchmuller and D. Wyler, Nucl. Phys. B268, 621 (1986).

[28] C. N. Leung, S. T. Love, and S. Rao, Z. Phys. C 31, 433 (1986).

[29] A. De Rujula, M. B. Gavela, P. Hernandez, and E. Masso, Nucl. Phys. B384, 3 (1992).

[30] K. Hagiwara, S. Ishihara, R. Szalapski, and D. Zeppenfeld, Phys. Rev. D 48, 2182 (1993).

[31] M. C. Gonzalez-Garcia, Int. J. Mod. Phys. A 14, 3121 (1999).

[32] B. Grzadkowski, M. Iskrzynski, M. Misiak, and J. Rosiek, J. High Energy Phys. 10 (2010) 085.

[33] G. Passarino, Nucl. Phys. B868, 416 (2013).

[34] E. da Silva Almeida, N. Rosa-Agostinho, O. J. Éboli, and M. Gonzalez-Garcia, Phys. Rev. D 100, 013003 (2019). 
[35] J. de Blas, M. Ciuchini, E. Franco, S. Mishima, M. Pierini, L. Reina, and L. Silvestrini, Proc. Sci., EPS-HEP2017 (2017) 467 [arXiv:1710.05402].

[36] A. Falkowski and D. Straub, J. High Energy Phys. 04 (2020) 066.

[37] S. Dawson, S. Homiller, and S. D. Lane, Phys. Rev. D 102, 055012 (2020).

[38] Report on the Physics at the HL-LHC, and Perspectives for the HE-LHC, edited by A. Dainese, M. Mangano, A. B. Meyer, A. Nisati, G. Salam, and M. A. Vesterinen, Vol. 7/ 2019 of CERN Yellow Reports: Monographs (CERN, Geneva, Switzerland, 2019), ISBN 978-92-9083-549-3.
[39] T. Corbett, O. Éboli, and M. Gonzalez-Garcia, Phys. Rev. D 96, 035006 (2017).

[40] R. D. Ball, V. Bertone, F. Cerutti, L. Del Debbio, S. Forte, A. Guffanti, J. I. Latorre, J. Rojo, and M. Ubiali (NNPDF Collaboration), Nucl. Phys. B855, 153 (2012).

[41] A. Buckley, J. Ferrando, S. Lloyd, K. Nordstrm, B. Page, M. Rfenacht, M. Schnherr, and G. Watt, Eur. Phys. J. C 75, 132 (2015).

[42] A. Alloul, N. D. Christensen, C. Degrande, C. Duhr, and B. Fuks, Comput. Phys. Commun. 185, 2250 (2014).

[43] J. Alwall, M. Herquet, F. Maltoni, O. Mattelaer, and T. Stelzer, J. High Energy Phys. 06 (2011) 128. 